\title{
Forum
}

doi: $10.1017 / \mathrm{S} 0373463310000536$

\section{A New Method For DGPS Ambiguity Resolution?}

\author{
P.J.G. Teunissen \\ (GNSS Research Centre, Curtin University of Technology, Perth) \\ (Email: p.teunissen@curtin.edu.au) \\ KEY WORDS \\ 1. Integer Ambiguity Resolution. 2. LAMBDA Method. 3. Ambiguity Success Rate.
}

1. INTRODUCTION. The Journal of Navigation recently published a paper [29] in which it is claimed that a new integer ambiguity resolution method, inspired by the LAMBDA method [1], is developed and presented. Unfortunately, this paper, here referred to as Piergentili, contains serious errors and misconceptions about integer ambiguity resolution (IAR). This becomes clear once one has a proper understanding of the existing body of knowledge in the field of GNSS ambiguity resolution.

2. THEORY OF INTEGER INFERENCE. The theory that underpins ultra-precise GNSS parameter estimation is the theory of integer inference [2, 3]. Central in the theory of integer inference is the mixed integer model. It is defined as:

$$
\mathrm{E}(y)=A a+B b, a \in \mathbb{Z}^{n}, b \in \mathbb{R}^{p},
$$

in which the $m$-vector $y$ contains the 'observed minus computed' pseudo ranges and carrier-phase observables, E denotes the mathematical expectation, $(A, B)$ is the $m \times(n+p)$ design matrix, the $n$-vector $a$ contains the integer double-differenced ambiguities and the real-valued $p$-vector $b$ contains the remaining unknown parameters, such as baseline components (coordinates) in the case of the geometry-based model or receiver-satellite ranges in the case of the geometry-free model, and possibly atmospheric delay parameters (troposphere, ionosphere). The latter parameters may be assumed absent in the case of sufficiently short baselines. Matrix $A$ contains the wavelength(s) and matrix $B$ is formed, in the case of the geometry-based model, from the receiver-satellite direction vectors; it thus contains the relative receiversatellite geometry information.

The mixed integer model is solved in three steps [1]. First, the integer nature of $a$ is discarded and the parameters $a$ and $b$ are estimated using the principle of (weighted) least-squares (LS) estimation. This gives the so-called float solutions of the ambiguities and baseline, $\hat{a}$ and $\hat{b}$, together with their variance matrices $Q_{\hat{a} \hat{a}}$ and $Q_{\hat{b} \hat{b}}$, 
and covariance matrix $Q_{\hat{b} \hat{a}}$. Then the integer ambiguity constraints are taken into account by a mapping $I$ of $\hat{a}$ :

$$
\breve{a}=\mathrm{I}(\hat{a}) .
$$

Finally in the third step, the resolved ambiguity vector $\breve{a}$ is used to re-adjust the float estimator $\hat{b}$, so as to obtain the fixed baseline estimator:

$$
\breve{b}=\hat{b}-Q_{\hat{b} \hat{a}} Q_{\hat{a} \hat{a}}^{-1}(\hat{a}-\breve{a})
$$

The mapping I of Equation (2) can be defined in many different ways. The following three classes of such estimators exist [3]: the class of integer estimators (I), the class of integer aperture estimators (IA), and the class of integer equivariant estimators (IE). They are subsets of one another and related as:

$$
\mathrm{I} \subset \mathrm{IA} \subset \mathrm{IE} .
$$

Each class consists of a multitude of ambiguity estimators and each class has its own optimal estimator. Optimality refers here to the maximization of the probability of correct integer estimation (the success rate) or to the minimization of the mean squared error. The three optimal estimators, one for each class, have been identified in [4-6].

Of the three classes, I, IA and IE, the class of integer estimators is most often used. Within this class, the three most popular estimators are integer rounding (IR), integer bootstrapping (IB), and integer least-squares (ILS). In $[4,7]$ it is proven that their success-rates are related as:

$$
\mathrm{P}\left(\breve{a}_{\mathrm{IR}}=a\right) \leqslant \mathrm{P}\left(\breve{a}_{\mathrm{IB}}=a\right) \leqslant \mathrm{P}\left(\breve{a}_{\mathrm{ILS}}=a\right)
$$

Thus integer rounding (IR) has the poorest performance, while integer least-squares (ILS) has the best performance.

3. LAMBDA METHOD. The LAMBDA method, introduced in [1], is a computationally efficient mechanization of the ILS principle. It therefore produces ambiguity solutions with the highest possible success rate. Hence, on the basis of the same underlying assumptions, no other integer ambiguity estimator exists that can produce higher success rates than LAMBDA. The computational efficiency of the method is for a large part due to the method's decorrelating ambiguity transformation. It transforms the usually extremely elongated double differenced ambiguity search space to a more spherical shape, thus enabling a much more efficient integer ambiguity search.

The LAMBDA method applies to any mixed integer model (1). It is therefore application independent. It can and has been used for single-frequency and multifrequency models, for single-epoch and multi-epoch models, and for current and future GNSS, stand alone or in combination. It has also been used in disciplines other than GNSS. Examples are radar remote sensing [8], VLBI [9] and acoustic marine positioning [10]. Details of the method can be found in [1, 11]. An elementary description is given in $[12,13]$, see also the FAQs of [14]. (note: Piergentili refers to [13], but gives a wrong reference). 
4. PIERGENTILI'S IAR METHOD. With the above theoretical background, one may ask how the work of Piergentili relates to this existing body of knowledge? Although Piergentili's method of IAR (c.f. Equation 7) is presented as an ILS technique, it is essentially based on integer rounding, because Piergentili's objective function is unfortunately chosen to be univariate (in the scalar case, ILS, IB and IR are identical). We will come back to this in a moment.

We start with Piergentili's motivation for developing an IAR method in the first place. In the paper it is stated that the LAMBDA method 'is applicable only in postprocessing analysis of data'. This statement is false. In the more than 15 year existence of the method, it has been successfully used in real-time by many, in a variety of different and demanding applications. Some such examples are real-time (automatic) aircraft landings $[15,16]$, real-time relative navigation of spacecraft $[17,18]$, and realtime attitude determination $[19,20]$. The method is also widely used in industry and by receiver manufacturers, for instance, in Leica's SpiderNET [21], in Septentrio receivers [22] and in the satellite compasses of Furuno [23].

Piergentili's statement that LAMBDA's 'convergence time is quite long' is also wrong. In fact, upon its introduction, LAMBDA made epoch-by-epoch or instantaneous GNSS positioning possible. For examples, see [24-27]. Finally, the statement that the 'Wide Lane technique is faster' than LAMBDA, is also incorrect. In [28] it has been shown that the wide lane combination is a special case of LAMBDA's automated decorrelation step.

Now let us turn to Piergentili's IAR method itself. In the introduction it is stated that 'the number of satellites used for DGPS should be kept low to guarantee brief calculation time'. Although this point is not further clarified in the paper, it is generally a counterproductive strategy from an IAR point of view. The more satellites are tracked, the higher the ambiguity success rate generally is for such short baselines as treated in the paper. That Piergentili's method is indeed not able to realize high success rates in short time, is in fact acknowledged in the paper's statement (p. 650) that it needs 'a period of two minutes for GV evaluation' and 'a period of about twenty minutes for ambiguity evaluation'. Despite the paper's goal to enable fast ambiguity resolution, this is not really a fast initialization time. The reason lies mainly in Piergentili's unfortunate scalar approach (c.f. Equation 7), where IAR is done on an individual ambiguity by ambiguity basis. Since ILS is identical to IR in the scalar case, integer minimizing Piergentili's ambiguity objective function (c.f. Eq. 7) is identical to rounding its scalar float LS solution to its nearest integer. Hence, since in Piergentili's case the solution is constrained to a subset of integers, the solution is the subset integer which is nearest to the scalar float solution. Thus if the nearest integer is not in the subset, the next nearest integer is checked, etc. In this way, one alternates around the float solution until the evaluated integer is a member of the given subset.

It follows from the theory of integer inference that this nearest integer based scalar approach has two important drawbacks. First, the scalar approach implies that not all available information is taken into account. The correlation between the ambiguities, for instance, is not taken into account. More importantly however, the relative receiver-satellite geometry is also not taken into account, thus effectively making Piergentili's method a geometry-free method [2], with corresponding significantly reduced strength of successful IAR. Secondly, since the ambiguities are resolved on an individual scalar basis, the absence of a vectorial formulation implies 
that no advantage can be taken of the optimal ILS success rate performance, see Equation (5).

It is rather unfortunate, given Piergentili's claim of a new method, that the paper has not properly explored the existing body of knowledge. With such a conceptual and numerical comparison, the errors and misconceptions could have been avoided.

\section{REFERENCES}

[1] Teunissen PJG (1995): The least-squares ambiguity decorrelation adjustment: a method for fast GPS integer ambiguity estimation. Journal of Geodesy, 70, 65-82.

[2] Teunissen PJG (1997): A canonical theory for short GPS baselines. Part I: The baseline precision, Part II: the ambiguity precision and correlation, Part III: the geometry of the ambiguity search space, Part IV: precision versus reliability. Journal of Geodesy, 71: 513-525, 389-401, 486-501, 320-336.

[3] Teunissen PJG (2003): Towards a unified theory of GNSS ambiguity resolution. Journal of Global Positioning Systems, 2(1), 1-12.

[4] Teunissen PJG (1999): An optimality property of the integer least-squares estimator. Journal of Geodesy, 73, 587-593.

[5] Verhagen AA, PJG Teunissen (2006): New global navigation satellite system ambiguity resolution method compared to existing approaches. Journal of Guidance, Control, and Dynamics, 29(4), 981-991.

[6] Teunissen PJG (2003): Theory of integer equivariant estimation with application to GNSS, Journal of Geodesy, 77, 402-410.

[7] Teunissen PJG (1998): Success probability of integer GPS ambiguity rounding and bootstrapping. Journal of Geodesy, 72, 606-612.

[8] B. M. Kampes, R. F. Hanssen (2004), "Ambiguity Resolution for Permanent Scatterer Interferometry", IEEE Transactions On Geoscience And Remote Sensing, vol. 42, no. 11, 2446-2453.

[9] Hobiger T, M Sekido, Y Koyama, T Kondo (2008): Integer phase ambiguity estimation in nextgeneration geodetic Very Long Baseline Interferometry. J. Adv. Space Res., doi:10.1016/j.asr. 2008.06.004.

[10] Viegas D, S Cunha (2007): Precise Positioning by Phase Processing of Sound Waves. IEEE Trans on Signal Processing, Vol. 55, No. 12, 5731-5738.

[11] de Jonge PJ, CCJM Tiberius (1996): The LAMBDA method for integer ambiguity estimation: implementation aspects. Delft Geodetic Computing Centre, LGR report No. 12, Delft, The Netherlands.

[12] Teunissen PJG, PJ de Jonge, CCJM Tiberius (1995): A new way to fix carrier-phase ambiguities. Innovation, GPS World, April 1995, 58-61.

[13] Teunissen PJG, PJ de Jonge, CCJM Tiberius (1995): The LAMBDA method for fast GPS surveying. Proceedings International Symposium "GPS Technology Applications" Bucharest, Romania, Sept. 26-29, 1995, 1-8.

[14] Joosten P, CCJM Tiberius (2002): LAMBDA: FAQs. GPS Solutions 6: 109-114.

[15] Boon F, BAC Ambrosius (1997): Results of real-time application of the LAMBDA method in GPS based aircraft landings. Proc. KIS97, 339-345.

[16] Waters J, P Sousa, L Wellons, G Colby, J Weir (2001): Test Results of an F/A-18 Automatic Carrier Landing Using Shipboard Relative GPS. ION 57th Annual Meeting/CIGTF 20th Biennial Guidance Test Symposium 11-13 June 2001, Albuquerque, 841-851.

[17] Cox DB, JD Brading (2000): Integration of LAMBDA ambiguity resolution with Kalman filter for relative navigation of spacecraft. Navigation, 47(3), 205-210.

[18] Wu SC, YE Bar-Server (2006): Real-time, sub-cm differential orbit determination of two low-earth orbiters with GPS bias-fixing. Proc. ION-GNSS, 2515-2522.

[19] Dai L, KV Ling, N Nagarajan (2004): Real-time attitude determination for Microsatellite by LAMBDA method combined with Kalman filtering. Proc. AIAA-ICSS 2004, 3118, 1-8. 
[20] Monikes R, O Meister, J Wendel, GF Trommer (2006): Yaw-angle estimation of the VTOL-UAVs with the extended LAMBDA method and low cost receivers. Proc ION-NTM 2007, 179-186.

[21] Leica Geosystems (2005): Networked reference stations; take it to the Max. White paper, 11p.

[22] Van der Kuylen I, P Nemry, F Boon, A Simsky (2006): Comparison of attitude performance for multi-antenna receivers. European Journal of Navigation, 4(2), 1-9.

[23] Furuno (2007): Satellite Compass SC-20, SC-50, SC-110. furuno.co.jp/marine products.

[24] Teunissen PJG, PJ de Jonge, CCJM Tiberius (1997): Performance of the LAMBDA method for fast GPS ambiguity resolution, Navigation, Journal of the Institute of Navigation, Vol. 44, No. 3, Fall 1997, 373-383.

[25] Bock Y, RM Nikolaidis, PJ de Jong (2000): Instantaneous geodetic positioning at medium distances with the Global Positioning System. Journal of geophysical research, Vol. 105, B12, 28, 223-28, 253.

[26] Ji S, W Chen, C Zhao, X Ding, Y Chen (2007): Single-epoch ambiguity resolution for Galileo with the CAR and LAMBDA methods. GPS Solutions,

[27] Hernandez-Pajares M et al. (2010): Wide Area RTK: High precision positioning on a continental scale. Inside GNSS, March/April 2010, 35-46.

[28] Teunissen PJG (1997): On the GPS widelane and its decorrelating property. Journal of Geodesy, 71, 577-587.

[29] Piergentili F, Cordelli E (2010): A New Method for DGPS Ambiguity Resolution. The Journal of Navigation 63, 645-661. 